\title{
Improved grey-correlation mode for analyzing influence of landslide factors at slope stability
}

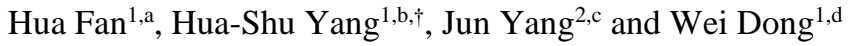 \\ ${ }^{1}$ Kunming University of Science and Technology, Kunming 650051, China \\ ${ }^{2}$ China Construction Steel Structure Co., Ltd. , Chongqing 400045, China \\ *Corresponding author: Hua-Shu YANG. E-mail: yhs005914@qq.com
}

\begin{abstract}
Conventional grey theory has flaws for analysing landslide factors, so grey-correlation was improved by multianalysis, in order to availably analyse sensitivity of landslide factors on slope stability. Then worst factor would be found out for slope stability, and engineering reinforcement would have preferable pertinence. The dissimilarities and merits of improved calculating mode were discussed with conventional grey theory. Analysis on landslide instances pointed out: influencing factor's sensitivity by improved calculating accords more with factual cases of landslide, which would be used for security checkup of precarious slope and emphasizing particularly on dangerous factor. And this disquisition would be used for reference by pursuers at realms like structured materials and reliability assessment etc.
\end{abstract}

Keywords: Reliability assessment; Landslide factors; Sensitivity analysis; Grey-correlation theory.

\section{Introduction}

In early the 80's of 20 century, Ju-Long DENG put forward gray system theory, and gray correlation analysis has been applied to various fields, and has achieved fruitful results up to now. The application of gray correlation method in stability analysis of landslide has been gradually paid more attention. Such as Zhi-Bin LI introduced gray model to evaluate the risk of landslide and debris flow [1], Yang WANG and others analyzed sensitivity factors the landslide based on gray correlation model[2], Jun-Qing WANG and others discussed sensitivities of main factors at loessial high slope based on gray correlation model[3].

Generally, in order to make different dimension or different magnitude, the index would be compared, the data would be normalized, then the correlation degree could be calculated. Obtained correlation is only a relative contrast value, but the actual order of magnitude is often neglected, and it is easy to cause distortion results. With the cohesion of landslide and influence the average unit weight on landslide as an example, the cohesion turned to be $0.5 \mathrm{a}$ from a, causing 
stability coefficient changed from $0.9 \mathrm{~K}$ to $\mathrm{K}$. If solve by the traditional methods, and normalized the calculation results, the correlation coefficient obtained are the same, and deduce the same sensitivity. And the result is contrast to the analysis of actual numbers. Therefore, the calculation model of gray correlation needs to be further improved for analysing main landslide factors of structured materials.

Considering above problems, combined with un-unitary idea, gray correlation model was improved, then sensitivity of each landslide factor was analyzed, so worst factors was worked out for slope stability.

\section{Improving Processes}

Grey relation analysis is based on sequence changes of time data, which is important methods to study correlation of multifarious things. The basic idea is to judge the correlation degree by determining the geometric similarity degree the reference data series the target and factor variable data column, the quantitative method are used to confirm the influence degree of factors on target. The shapes the two curves are more similar, the larger the correlation degree between them is; on contrary, the smaller the correlation degree is. This method is an intuitive method, which evaluates factor sensitivity by virtue of measuring correlation degree between this factor and stability coefficient of slope.

\subsection{Arithmetic Equations of Grey-correlation}

The Sequence Matrices. A reference sequence was set to $X=\left[X_{1}, X_{2}, \ldots, X_{i}, \ldots\right.$, $\left.X_{m}\right]^{T}$

and comparison matrix was $Y=\left[Y_{1}, Y_{2}, \ldots, Y_{i}, \ldots, Y_{m}\right]^{T}$.

Hereinto $X_{i}=\left\{x_{i}(1), x_{i}(2), \ldots, x_{i}(j), \ldots, x_{i}(n)\right\} ; Y_{i}=\left\{y_{i}(1), y_{i}(2), \ldots, y_{i}(j), \ldots\right.$, $\left.y_{i}(n)\right\}$.

Nondimensional Modeling. Multifarious parameters induced landslide are not the same, and the difference at order of magnitude is very big, so cannot be directly compared. If we want to calculate directly, wrong conclusion would easily be got or the calculation would be very complicated. So nondimensional treatment should be used for raw data. Dimensionless methods involve: center, range, maximum value, minimum value and average etc. On base of comparing and studying, the range method was adopted for analysing landslide parameters, shown in Eq.1. 


$$
\left.\begin{array}{rl}
x_{i}^{\prime}(j) & =\frac{x_{i}(j)-\min \left\{X_{i}\right\}}{\max \left\{X_{i}\right\}-\min \left\{X_{i}\right\}} \\
y_{i}^{\prime}(j) & =\frac{y_{i}(j)-\min \left\{Y_{i}\right\}}{\max \left\{Y_{i}\right\}-\min \left\{Y_{i}\right\}}
\end{array}\right\} .
$$

Correlation Coefficients. Grey-correlation coefficient was replaced by $\xi_{i}(j)$ on comparison matrix and reference sequence was the point of KTH. Grey-correlation coefficients were calculated by classical calculation method of Deng[4]:

$$
\left.\begin{array}{l}
\Delta_{\max }=\max _{i} \max _{j}\left|x_{i}^{\prime}(j)-y_{i}^{\prime}(j)\right| \\
\Delta_{\min }=\min _{i} \min _{j}\left|x_{i}^{\prime}(j)-y_{i}^{\prime}(j)\right| \\
\xi_{i}(j)=\frac{\Delta_{\min }+\rho \Delta_{\max }}{\left|x_{i}^{\prime}(j)-y_{i}^{\prime}(j)\right|+\rho \Delta_{\max }}
\end{array}\right\} .
$$

In Eq. 2, $\rho$ is resolution coefficient and $\rho \in(0,1)$. Usually $\rho$ is taken as 0.5 , and in this paper, 0.5 was taken as the value of $\rho$.

Sensitivities of Landslide Factors. Correlation coefficient is the value of correlation degree at each point in the curve, but test data and actual information are too scattered to carry on study and comparison as a whole. So mean values $\left(r_{i}\right)$ of correlation degree were used for showing sensitivities of factors, and arithmetic was shown in Eq.3.

$$
r_{i}=\frac{1}{m} \sum_{j=1}^{m} \xi_{i}(j)
$$

\subsection{Modified algorithm}

First of all, adopt improved method of grey relational degree for importance answers at landslide factors, the improved method was proposed by Jie CUI etc[5]. Then determine weightings of those factors, the algorithm is as follows.

Weighting coefficients $\left(\xi_{0}\right)$ for common reference were shown in Eq.4. 


$$
\xi_{0}(j)=\max \left\{\xi_{1}(j), \xi_{2}(j), \cdots, \xi_{m}(j)\right\}
$$

Differences $\left(D_{i}\right)$ between index-correlation's data and $\xi_{0}$ were shown in Eq.5.

$$
D_{i}(j)=\sum_{j=1}^{m}\left(\xi_{0}^{\prime}(j)-\xi_{i}(j)\right)^{2}
$$

Calculate Weightings $\left(w_{i}\right)$ of each factor inducing landslide were shown in Eq.6.

$$
w_{i}=\frac{1}{1+D_{i}} \text {. }
$$

Then sensitivity coefficients $\left(S_{i}\right)$ could be calculate by Eq.7 [6]:

$$
S_{i}=\left(\max \left\{X_{i}\right\}-\min \left\{X_{i}\right\}\right) \times w_{i}
$$

Value $\left(S_{i}\right)$ represents important degree of inspecting landslide factor, and the value $\left(S_{i}\right)$ is bigger, the affecting force is bigger on slope stability when this factor changing.

\section{Actual Example of Reliability Assessment at Slope Stability}

\begin{tabular}{|c|c|c|c|c|c|c|c|c|c|c|c|}
\hline $\begin{array}{c}c \\
{[\mathrm{kPa}]}\end{array}$ & $S C$ & $\stackrel{\varphi}{\varphi}\left[{ }^{\circ}\right]$ & $S C$ & $\begin{array}{c}\boldsymbol{G} \\
{\left[\mathrm{kN} / \mathrm{m}^{3}\right]}\end{array}$ & $S C$ & $\begin{array}{c}M \\
{[\%]}\end{array}$ & $S C$ & $\begin{array}{c}\boldsymbol{H} \\
{[\mathrm{m}]}\end{array}$ & $S C$ & $\begin{array}{c}\boldsymbol{R} \\
{[\mathrm{h}]}\end{array}$ & $S C$ \\
\hline 40 & 0.935 & 6 & 0.726 & 18.1 & 1.03 & 49 & 1.15 & 50 & 1.058 & 8 & 1.016 \\
\hline 45 & 0.962 & 7.5 & 0.822 & 18.6 & 1.026 & 64 & 1.094 & 60 & 1.047 & 16 & 1.016 \\
\hline 50 & 0.989 & 9 & 0.919 & 19.1 & 1.021 & 81 & 1.024 & 70 & 1.037 & 24 & 1.016 \\
\hline 55 & 1.016 & 10.5 & 1.016 & 19.6 & 1.016 & 100 & 1.016 & 80 & 1.016 & 0 & 1.016 \\
\hline 60 & 1.043 & 12 & 1.115 & 20.1 & 1.012 & 121 & 0.979 & 90 & 0.982 & 32 & 1.016 \\
\hline 65 & 1.071 & 13.5 & 1.214 & 20.6 & 1.008 & 144 & 0.954 & 100 & 0.939 & 40 & 1.014 \\
\hline 70 & 1.098 & 15 & 1.315 & 21.1 & 1.004 & 169 & 0.923 & 110 & 0.886 & 48 & 1.007 \\
\hline
\end{tabular}

The Example locates Malipo county in Yunnan province, China. When landslide factors were solely changed, stability coefficient $(S C)$ changed based on test data, as shown in Table 1. 
Symbolic meanings were defined as follows: $c$ - cohesion of slope materials; $\varphi$ - internal friction angle; $G$ - unit weighting; $M$ - scale of mathematical model, prototype' sizes of actual example are 159m (height) and 338m (aclinic length); $H$ - seeping water head in slope; $R$ - duration of rainfall.

\subsection{Calculating for correlative degrees}

To apply Eq. 1 and Eq. 2 based on data of Table 1, multifarious grey-correlation coefficients were calculated and were shown in Table 2.

To apply Eq. 3 based on data of Table 2, mean values of correlation degree were calculated and were shown in Table 3. To apply Eq. 1 and Eq. 4 - Eq. 7 based on data of Table 2, modified sensitivity coefficients were calculated and were shown in Table 3.

For slope stability of this example, the forces of landslide factors can be seen from Table 3. For conventional mode, forces order are $c>\varphi>M>G>H>R$, but forces order are $\varphi>c>M>H>G>R$ for improved mode.

Table 2. Grey-correlation coefficient $(\xi)$ between landslide factors and slope stability

\begin{tabular}{|c|c|c|c|c|c|c|c|c|c|c|c|}
\hline $\begin{array}{c}c \\
{[\mathrm{kPa}]}\end{array}$ & $\xi$ & $\begin{array}{c}\varphi \\
{[\circ]}\end{array}$ & $\xi$ & $\begin{array}{c}\boldsymbol{G} \\
{\left[\mathrm{kN} / \mathrm{m}^{3}\right]}\end{array}$ & $\xi$ & $\begin{array}{c}M \\
{[\%]}\end{array}$ & $\xi$ & $\begin{array}{c}\boldsymbol{H} \\
{[\mathrm{m}]}\end{array}$ & $\xi$ & $\begin{array}{c}\boldsymbol{R} \\
{[\mathrm{h}]}\end{array}$ & $\xi$ \\
\hline 40 & 1 & 6 & 1 & 18.1 & 0.33 & 0.49 & 0.38 & 50 & 0.33 & 8 & 0.38 \\
\hline 45 & 1 & 7.5 & 0.99 & 18.6 & 0.42 & 0.64 & 0.5 & 60 & 0.39 & 16 & 0.43 \\
\hline 50 & 1 & 9 & 0.99 & 19.1 & 0.61 & 0.81 & 0.83 & 70 & 0.48 & 24 & 0.5 \\
\hline 55 & 0.99 & 10.5 & 0.98 & 19.6 & 0.93 & 1 & 0.97 & 80 & 0.66 & 0 & 0.33 \\
\hline 60 & 0.99 & 12 & 0.99 & 20.1 & 0.58 & 1.21 & 0.66 & 90 & 0.82 & 32 & 0.6 \\
\hline 65 & 1 & 13.5 & 0.99 & 20.6 & 0.42 & 1.44 & 0.52 & 100 & 0.49 & 40 & 0.9 \\
\hline 70 & 1 & 15 & 1 & 21.1 & 0.33 & 1.69 & 0.43 & 110 & 0.33 & 48 & 0.33 \\
\hline
\end{tabular}

Table 3. Contrast conventional mode with improved mode at grey-correlation

\begin{tabular}{ccccccc}
\hline Algorithm & $\boldsymbol{c}$ & $\boldsymbol{\varphi}$ & $\boldsymbol{G}$ & $\boldsymbol{M}$ & $\boldsymbol{H}$ & $\boldsymbol{R}$ \\
\hline Conventional mode & 0.997 & 0.992 & 0.519 & 0.612 & 0.501 & 0.496 \\
Improved mode & 0.163 & 0.589 & 0.009 & 0.097 & 0.059 & 0.003 \\
\hline
\end{tabular}

\subsection{Analysis at calculatingly results}

One of Differences in Table 3. The difference of relation values is wee on cohesion $(c)$ and friction angle $(\varphi)$ calculated by conventional mode. It is difficult to distinguish which the two factors the Internal friction angle and cohesive force are more sensitivity to the influence the landslide. But the average correlation coefficients are all above 0.97 , which indicates that factor of landslide is wonderful, and it represents to be a linear relationship. There may be two main reasons to cause this phenomenon as follows.

In Long-term research result, internal friction angle and cohesive force are regarded as key factor of affecting stability on slope, so $c$ and $\varphi$ are strongly 
correlated with slope stability. Secondly, the multiple gray correlation researches above are all based on output the software simulation, the landslide shear strength Eq. appears to be a linear relationship both with the internal friction angle and cohesion in the calculation, causing the great relevance. The first factor only aims at a few landslides; it cannot be applied to most the analysis the landslide. Therefore, the second reason is more reasonable to explain the phenomenon in this analysis. the correlation with slope stability is very big and very close which is caused by the internal friction angle and cohesion.

Comparing conventional methods with improved method, mainly difference is order of $c, \varphi$ and $G, H$ in slope. We can see clearly from the Table 1 that the greatest influence the internal friction angle has on stability coefficient can reach to 0.589 , significantly higher than 0.163 the biggest influence the cohesion has on stability coefficient. Field investigation and indoor experiment found that the cohesion fully weathered rocks had reached to $55 \mathrm{kPa}$, and the internal friction angle is only $10.5^{\circ}$, however, the unstable factor that the landslide are easy to cause disruption are determined by sensitivity factor the weakest link. Cohesion and internal friction angle are the main disrupting factors of glide plane, it should be the major sensitivity factors, while the internal friction angle is obviously lower than that the same cohesion level, namely, the internal friction angle is more sensitivity to the sliding failure than the cohesion. Therefore, the internal friction angle is the most sensitivity factor which can influence stability of slope, cohesion is sensitivity factor which is inferior to internal friction angle. It is consistent with theoretical analysis, computer simulation, and experimental results, actual phenomenon.

Secondary Difference in Table 3. Obvious difference between the improved method and the conventional method is that the influences of gravity and water are different on slope stability. The mechanism that the rock unit weight affects the landslide is mainly that the gravity the landslide body increases with the increase the gravity of rock unit weight, the slide force under the potential sliding surface also increased with it, make the slip plane change. And the main cause that the landslide gravity change is that the landslide material turned to be saturated unit weight from the natural unit weight due to the changes in water content, making the landslide slip force increase, and slope changes. Of course, perpendicular pressure above sliding surface (anti-sliding force) would increase along with unit weight of landslide mass, so sliding action with unit weight would be weaken to landslide.

Groundwater influences slope stability by virtue of changing material properties principally. If potential sliding surface of slope is affected by the groundwater, water content of rock mass would be increased, and internal friction 
angle and cohesion in material would fall rapidly, making geologic hazard happen much more easy. Water level' change has a greater influence on landslide as to unit weight, therefore sensitivity coefficient $(H)$ is bigger than sensitivity coefficient $(G)$ in Table 3, and it more conforms to theory analyse and the actual situation. Generalizing above, improved gray-correlation method is more consistent with objectivity reality.

\section{Conclusions}

For the sake of effect and profit on reinforcement engineering, the worst factor might inducing landslide has to be found. Improved equation was put out based on grey-correlation theory, which aimed at conventional mode' flaws on analysing landslide factors.

Based on test data, numerical simulation on slope was actualized by seepage theory and GeoStuido, so influence values on slope stability was gotten with changes of multifarious landslide factors. Importance order of multifarious landslide factors was analysed between correlation coefficient and sensitivity coefficient at slope stability, adopting conventional mode and improved mode respectively.

Analytical result on actual landslide pointed out: improved mode' resolving power is clearly better than conventional mode' when ordering importance of cohesion $(c)$ and friction angle $(\varphi)$ in slope material, to induce landslide. And improved mode's verdict accord with the fact much more than conventional mode' when ordering importance of multifarious landslide factors.

For slope stability related to example, adopting calculation result of improved grey-correlation mode, importance order of hexad landslide factors is: $\varphi>c>M>H>G>R$. So slurry penetration should be used for reinforcing internal friction angle and cohesion in slope material, which being more provided with pertinence at landslide.

\section{Acknowledgement}

This work was financially supported by The National Natural Science Foundation of China (Grant No.41462013, No.50869003, No.51069003), and by Scientific Research Foundation of Education Department of Yunnan Province, China (Grant No. 2015Y072).

Corresponding author: Yang Huashu, professor, doctoral tutor, and a academic leaders of Yunnan Province, China. E-mail: yhs005914@qq.com. 


\section{References}

[1]. Zhi-Bin LI, Cheng-De, ZHENG. Grey-mode identification and model for risk degree at landslide and debris flow, J. Systems Engineering-theory \& Practice. 5 (2000) 128-132. (in Chinese)

[2]. Yang WANG, Kun-Long YIN, Guan-Feng AN. Grey-correlation analysis of sensitivity factors of landslide, J. Rock and Soil Mechanics. 25 (2004) 91-93. (in Chinese)

[3]. Jun-Qing WANG, Jing LI, Qi, LI. Analysis of influence factors of high slope stability of loess: Taking the Baojixia Water Division Project for example, J. Rock and Soil Mechanics. 30 (2009) 2117-2118. (in Chinese)

[4]. Ju-Long, DENG. The Tutorial of Grey System Theory. Huazhong University of Technology Press, Wuhan of China, 1990. (in Chinese)

[5]. Jie CUI, Yao-Guo DANG, Si-Feng LIU. An improved approach for determining weightings of attributes in decision making based on grey incidence, J. Chinese Journal of Management Science. 16 (2008) 141-145. (in Chinese)

[6]. Zhi-Bo CHEN, Wen-Bin JAN. Sensibility analysis of slopes stability based on grey-correlation analysis, J. Journal of Disaster Prevention and Mitigation Engineering. 26 (2006) 473-477. (in Chinese) 